\title{
Deep survey of the Segue 1 dwarf spheroidal galaxy with the MAGIC Telescopes
}

\author{
Jelena ALEKSIĆ* \\ Institut de Física d'Altes Energies (IFAE), E-08193 Barcelona, Spain \\ E-mail: jelenalifae.es \\ Javier RICO \\ IFAE \\ E-mail: jricodifae.es

\section{Manel MARTINEZ} \\ IFAE \\ E-mail: martinez@ifae.es
}

\section{Saverio LOMBARDI}

Instituto Nazionale di Astrofisica, I-00136 Rome, Italy

E-mail: saverio.lombardiapd.infn.it

\section{on behalf of the MAGIC Collaboration}

\begin{abstract}
Discovering the nature of dark matter is one of the most exciting tasks of modern science. Among the targets suitable for dark matter searches, dwarf spheroidal galaxies are considered to be excellent candidates and, among them, Segue 1 stands out with mass-to-light ratio estimated to order of 1000. We present the results of the first stereoscopic observations of Segue 1 with MAGIC, carried out between 2011 and 2013. With almost 160 hours of good quality data, this is the deepest observational campaign on any dwarf galaxy by any Imaging Air Cherenkov Telescope so far. The analysis of the data is performed using a dedicated likelihood approach, optimized for signals with characteristic spectral features. We search for dark matter particles with mass in the 100 $\mathrm{GeV}-20 \mathrm{TeV}$ range, considering theoretical scenarios with different final state Standard Model particles, annihilation with internal bremsstrahlung and monochromatic line signals.
\end{abstract}

The European Physical Society Conference on High Energy Physics

18-24 July, 2013

Stockholm, Sweden

\footnotetext{
* Speaker.
} 


\section{Introduction}

The notion of dark matter (DM) has present for almost a century, but the question about its nature is still unanswered. Observational evidence and cosmological predictions indicate that DM represents almost $85 \%$ of the total matter content, and $\sim 25 \%$ of the total energy density of the Universe [1]. Discovering its essence is one of the most important tasks of modern science.

Nevertheless, despite strong efforts over the years, no experiment so far has been able to detect DM, directly or indirectly. The main focus of current searches is on DM composed of weakly interacting massive particles (WIMPs), that are of non-baryonic nature, thermally produced in the early Universe and stable on cosmological timescales. Furthermore, in order to have the correct relic density and to be in agreement with the Big Bang nucleosynthesis, WIMPs should also be cold - i.e. non-relativistic at the onset of the large structure formation. WIMPs are predicted to annihilate or decay into Standard Model (SM) particles, such as photons, that could be detected by the existing experiments. With the mass range predicted for these DM particles spaning from tens of $\mathrm{GeV}$ to few $\mathrm{TeV}$, those photons might be visible in the gamma ray domain, currently best explored by from space by the Large Area Telescope (LAT) on board the Fermi satellite [2], and from ground by the Imaging Air Cherenkov Telescopes (IACTs): H.E.S.S. [3], MAGIC [4] and VERITAS [5].

The gamma-ray flux from DM annihilation or decay that is to be measured on Earth is determined by two independent factors: one, coming from the particle physics and other, related to the astrophysics. The particle physics term is fixed for a given DM model and does not depend on the observed source. It describes the shape of the expected spectrum and gives the number of photons produced above a given energy threshold for the DM scenario in question. On the other hand, the astrophysical factor is determined by the DM density profile of a specific source and its distance.

The typical annihilation (decay) gamma-ray spectrum is predicted to be continuous and featureless, with photons mainly produced from the pion decay and the final state radiation of charged particles. Nevertheless, some distinctive spectral features could be present, like monochromatic line from direct DM annihilation (decay) into photons, or pronounced peak towards the kinematic limit from emission of virtual internal bremsstrahlung (VIB) photons.

The IACTs are observatories that deal with great variety of scientific objectives. The preference, however, is usually given to the astrophysical sources of conventional origin, whose spectral distributions are, in majority of cases, rather featureless and unknown in advance. Consequently, standard analysis tools and methods of IACTs are adapted for such signals, at the expense of sources whose emissions are predicted to contain some distinctive spectral features. Therefore, in this work, an alternative analysis approach is used instead: the full likelihood method is optimized for recognition of characteristic signatures DM interactions may leave in the spectrum [6]. Full likelihood takes maximal advantage of the spectral information, and almost solely through the inclusion of the a priori knowledge on the expected gamma-ray spectrum in the likelihood, it achieves significantly better sensitivity than the method currently used as standard in analysis chains of the IACTs.

Here are presented the results of DM searches in Segue 1 observations with MAGIC. First, the motivation behind the choice of Segue 1 as a suitable DM target is explained. This is followed by the details of the observations with MAGIC and subsequent full likelihood analysis. Lastly, results 
are interpreted in the light of various DM annihilation and decay scenarios.

\section{Segue 1 as the Dark Matter Candidate Source}

The dSphs galaxies are the most DM-dominated objects in the Universe known so far [7]. With mass-to-light ratio $(M / L)$ of order of 100 , or even $1000 M_{\odot} / L_{\odot}$, their relative closeness (up to $\sim 250 \mathrm{kpc}$ distance from Earth), and almost non-existent gamma-ray background (as there is no gas and no recent star formation), these systems are considered to be excellent targets for indirect DM searches. Furthermore, as baryons are the subdominant component in a dSph, and are not expected to significantly alter the DM density profile, studies of their stellar kinematics can be used to set relatively robust constraints on the values of the astrophysical factor, and by extension, to the DM flux [8].

Among the dSphs, the ultra-faint galaxy Segue 1 stands out with $M / L$ estimated to 3400 $M_{\odot} / L_{\odot}$ [9]. Furthermore, given its distance of only $\sim 23 \mathrm{kpc}$ and position in the Northern hemisphere outside of the Galactic plane, Segue 1 has been dubbed an excellent candidate source for DM searches with MAGIC. Its visibility from the telescope location is $\sim 370$ hours of dark time per year at low zenith angles.

\section{MAGIC Observations and Standard Analysis}

The Florian Goebel Major Atmospheric Gamma-ray Imaging Cherenkov (MAGIC) Telescopes are located at the Roque de los Muchachos Observatory $\left(28.8^{\circ} \mathrm{N}, 17.9^{\circ} \mathrm{W} ; 2200 \mathrm{~m}\right.$ a.s.1.) in the Canary island of La Palma, Spain. The system consists of two, $17 \mathrm{~m}$ diameter telescopes designed to have high sensitivity at low energies $(E<100 \mathrm{GeV})$. The first telescope, MAGIC-I, has been operational since 2004; in 2009, it was joined by MAGIC-II, and the instruments have been working in unison ever since. The trigger threshold of the system is $\sim 50 \mathrm{GeV}$ for the standard observations; the integral sensitivity above $250 \mathrm{GeV}$, for $5 \sigma$ detection in 50 hours, is $\sim 0.7 \%$ of the Crab Nebula flux, with an angular resolution better than $0.07^{\circ}[10]$.

Observations of Segue 1 were performed between January 2011 and February 2013, under dark night conditions, for zenith angle range between $13^{\circ}$ and $37^{\circ}$, thus ensuring the low energy threshold of the analysis. During this period, the telescopes underwent a series of significant hardware changes: first, at the end of 2011, the readout systems of both instruments were replaced by more advanced configurations, based on Domino Ring Sampler version 4 (DRS4) chips [11]; second, by the end of 2012 the camera of MAGIC-I was exchanged with a replica of that of MAGIC-II, with uniform pixel size and extended trigger area with respect to the old one [12]. As a result of the upgrade, the response of the telescopes varied throughout the Segue 1 campaign; therefore, the total data sample was split into four subsamples, each corresponding to a stable performance and response of the instruments. All of the subsamples were analyzed separately, with the use of contemporaneous Crab Nebula observations (for optimization of the analysis cuts and validation of the analysis procedures) and the dedicated Monte Carlo productions (for evaluation of the response functions). 
After the quality selection and data reduction procedures of the MAGIC standard analysis chain, the total observation time amounted to 157.9 hours of good-quality data, thus making these Segue 1 observations with MAGIC the longest exposure of any dSph galaxy by any IACT so far.

\section{Full Likelihood Analysis}

The Segue 1 observations were analyzed using the full likelihood approach, an analysis method optimized for recognition of some characteristic features in the spectrum, like those expected from DM annihilation and decay, by including them in the likelihood calculations [6]. The sensitivity gain achieved from the use of this approach ranges (depending on the signal model) is about a factor of 2 with respect to that of the so-called conventional method [13], currently standard for in the analyses of IACTs.

No significant gamma-ray excess was found above the background in the Segue 1 observations with MAGIC. Consequently, the $95 \%$ c.l. upper limits are set, on thermally averaged product of annihilation cross section and velocity, $\left\langle\sigma_{\mathrm{ann}} v\right\rangle$, and lower limits on DM particle lifetime, $\tau_{\chi}$, assuming different scenarios of DM annihilation and decay, respectively. In particular, the following channels of photon production from DM annihilation or decay are considered: secondary photons from SM final states, gamma-ray lines and VIB photons. The DM particle mass is taken to be in $100 \mathrm{GeV}-10 \mathrm{TeV}$ range for annihilation, and between $200 \mathrm{GeV}$ and $20 \mathrm{TeV}$ for decay scenarios. In order to make the study as model-independent as possible, for all of the channels the branching ratio is assumed to be $100 \%$. Furthermore, no additional boosts to the signal, either from presence of substructures, or from quantum effects were taken into the account.

Lastly, it should be mentioned that the results, presented in the following section, should be consider conservative: namely, constraint is set that the signal intensity is bound to the physical region during the full likelihood maximization - that is, the gamma-ray signal intensity is not allowed to take negative values. That way, negative fluctuations cannot produce artificially stringent limits.

\section{Results}

Here are summarized the results of DM searches in 157.9 hours of Segue 1 observations with MAGIC, for different considered photon production channels.

Secondary photons, from final state SM particles, lead to a continuous and rather featureless spectrum, with a cutoff at energy corresponding to the DM particle mass $\left(m_{\chi}\right)$. In this work, we consider photons from DM annihilation and decay into following channels: quark-antiquark $(b \bar{b}$, $t \bar{t})$, lepton-antilepton $\left(\mu^{+} \mu^{-}, \tau^{+} \tau^{-}\right)$and gauge boson pairs $\left(W^{+} W^{-}, Z Z\right)$. For the annihilation scenarios, the resulting upper limits on $\left\langle\sigma_{\mathrm{ann}} v\right\rangle$ are found to be of order $(1-6) \times 10^{-24} \mathrm{~cm}^{3} \mathrm{~s}^{-1}$. These are the strongest bounds from dSph observations by any IACT. Furthermore, limits for leptonic channels are even more stringent than the strongest Fermi-LAT ones, from the joint analysis of 10 dSphs [14], for $m_{\chi}$ above $300 \mathrm{GeV}$ and $450 \mathrm{GeV}$, for $\mu^{+} \mu^{-}$and $\tau^{+} \tau^{-}$channels, respectively. In addition, the constraints from this work are at least an order of magnitude stronger than the previous most sensitive MAGIC results, obtained from $\sim 30$ hours of Segue 1 observation with MAGIC-I only [15]. Regarding the decay into the final state channels, lower limits on $\tau_{\chi}$ are found to be of 
order $\sim\left(10^{25}-10^{26}\right) \mathrm{s}$ for the highest considered masses, $m_{\chi} \sim 20 \mathrm{TeV}$. This is compatible with the constraints estimated from the Fornax galaxy observations with H.E.S.S., but from one to two orders of magnitude less constraining (depending on the channel) than the bounds derived from Fermi-LAT isotropic gamma-ray data [16].

Search for monochromatic line is done assuming direct annihilation into two photons, or one photon and a $Z$ boson. Although these processes are highly loop suppressed, potential detection of line-like feature would provide a firm proof of DM existence, and also reveal some information regarding the nature of its particle. The corresponding upper limits on $\left\langle\sigma_{\mathrm{ann}} v\right\rangle$ from this work are of order $4 \times 10^{-26} \mathrm{~cm}^{3} \mathrm{~s}^{-1}$ for $\gamma \gamma$, and of $7 \times 10^{-26} \mathrm{~cm}^{3} \mathrm{~s}^{-1}$ for $Z \gamma$ final state (with $m_{\chi} \sim 250 \mathrm{GeV}$ ). These bounds are up to two orders of magnitude less constraining than the results from H.E.S.S. line searches in the central Galactic Halo [17], and one to two orders of magnitude weaker than the Fermi-LAT limits from the Galactic Center region [18]. This, however, is expected, given the different targets considered in these analyses. As for the line emission in the case of DM decay, the case of production of a photon and a neutrino is considered: the lower limit on $\tau_{\chi}$ is found to be of order $2 \times 10^{26} \mathrm{~s}$ for $m_{\chi} \sim 1.5 \mathrm{TeV}$.

Lastly, the contribution of VIB photons to the gamma-ray spectrum and their impact on detection prospects is considered. Assuming a fermionic DM particle, that couples to muons or tau leptons via Yukawa interactions with the scalar $\eta$, helicity suppression in the $s$-wave contribution to the $\left\langle\sigma_{\mathrm{ann}} v\right\rangle$ can be lifted by emission of a VIB photon. Depending on the mass-splitting parameter $\mu$, that is defined as the squared ratio of the masses of the scalar and DM particle, $\mu=\left(m_{\eta} / m_{\chi}\right)^{2}$, the VIB contribution to the spectrum is more or less pronounced [19]. We calculate the upper limits on $\left\langle\sigma_{\mathrm{ann}} v\right\rangle$ for $\mu^{+} \mu^{-}(\gamma)$ and $\tau^{+} \tau^{-}(\gamma)$ channels, for the most degenerate states $(1 \leq \mu \leq 2)$ for which the VIB contribution is the most pronounced. The obtained upper limits, for the muon channel, reach $\left\langle\sigma_{\mathrm{ann}} v\right\rangle \sim 9 \times 10^{-26} \mathrm{~cm}^{3} \mathrm{~s}^{-1}$ for $m_{\chi} \sim 250 \mathrm{GeV}$, and are similar for all the considered mass-splitting values. For $\tau^{+} \tau^{-}(\gamma)$, the degeneracy of the considered model is more relevant, and limits for extreme case when $\mu=1.05$ and the VIB peak is very pronounced $\left(\left\langle\sigma_{\mathrm{ann}} v\right\rangle \sim 3 \times 10^{-27} \mathrm{~cm}^{3} \mathrm{~s}^{-1}\right.$, are almost an order of magnitude more constraining than the ones for the case of less significant VIB contribution $(\mu=2.0)$. For both considered channels, estimated bounds are up to two - three orders of magnitude stronger than for the annihilation into the same final states, but without the VIB photons.

\section{Conclusions}

We report on indirect DM searches in dSph galaxy Segue 1 with MAGIC. Observations, carried out in stereo mode between January 2011 and February 2013, have resulted in 157.9 hours of good quality data, thus making this campaign the deepest survey of any dSph by any IACT so far.

The gathered data have been analyzed by means of the full likelihood method. Given that no significant gamma-ray signal was found, the limits are derived for annihilation and decay rates assuming various DM models. Those limits are the strongest bounds, for the considered channels, from dSph observations by IACTs, and, for some models and DM particle masses, also more constraining than the Fermi-LAT limits.

The acquired results represent an improvement, with respect to the previously most sensitive MAGIC DM searches, of an order of magnitude. This campaign is expected to continue in the 
following years, and to leave an important landmark in the field in the pre-Cherenkov Telescope Array [20] era.

Acknowledgments We would like to thank the Instituto de Astrofísica de Canarias for the excellent working conditions at the Observatorio del Roque de los Muchachos in La Palma. The support of the German BMBF and MPG, the Italian INFN, the Swiss National Fund SNF, and the Spanish MICINN is gratefully acknowledged. This work was also supported by the CPAN CSD2007-00042 and MultiDark CSD2009-00064 projects of the Spanish Consolider-Ingenio 2010 programme, by grant 127740 of the Academy of Finland, by the DFG Cluster of Excellence "Origin and Structure of the Universe", by the Croatian Science Foundation Project 09/176, by the DFG Collaborative Research Centers SFB823/C4 and SFB876/C3, and by the Polish MNiSzW grant 745/N-HESS-MAGIC/2010/0.

\section{References}

[1] P. A. R. Ade et al., (2013) [arXiv:1303.507].

[2] NASA Goddard Space Flight Center, http://fermi.gsfc.nasa.gov (September 2013).

[3] H.E.S.S. Collaboration, http://www.mpi-hd.mpg.de/hfm/HESS (September, 2013).

[4] MAGIC Collaboration, http://magic.mpp.mpg.de (September, 2013).

[5] VERITAS Collaboration, http://veritas. sao.arizona.edu (September 2013).

[6] J. Aleksić, J. Rico and M. Martinez, JCAP 10 (2012) 032 [arXiv: 1209.5589 ].

[7] L. E. Strigari et al., Astrophys. J. 678 (2008) 614-620 [arXiv: 0709.1510 ].

[8] N. Evans, F. Ferrer and S. Sarkar, Phys. Rev. D69 (2004) 123501 [arXiv : astro-ph/0311145].

[9] J. Simon et al., Astrophys. J. 733 (2011) 46-66 [arXiv: 1007 . 4198].

[10] J. Sitarek et al. In Proc. of the 33rd International Cosmic Ray Conference, Rio de Janeiro, Brazil (2013) [arXiv:1308.0141].

[11] J. Sitarek et al., Nucl. Instrum. Meth. A723 (2013) 109-120 [arXiv:1305 . 1007].

[12] D. Nakajima et al. In Proc. of the 33rd International Cosmic Ray Conference, Rio de Janeiro, Brazil (2013).

[13] W. A. Rolke, A. M. López and J. Conrad, Nucl. Instrum. Meth. A551 (2005) 493-503 [arXiv:physics/0403059].

[14] M. Ackermann et al., Phys. Rev. Lett. 107 (2011) 241302 [arXiv:1108.3546].

[15] J. Aleksić et al., JCAP 06 (2011) 035 [arXiv: 1103.0477 ].

[16] M. Cirelli et al., Phys. Rev. D86 (2012) 083506 [arXiv: 1205. 5283].

[17] A. Abramowski et al., Phys. Rev. Lett. 110 (2013) 041301 [arXiv: 1301.1173 ].

[18] M. Ackermann et al., Phys. Rev. D86 (2012) 02200 [arXiv: 1205. 2739].

[19] T. Bringmann et al., JCAP 07 (2012) 054 [arXiv: 1203.1312 ].

[20] The CTA Consortium, https://www. cta-observatory - org (September 2013). 